\title{
Miguel de Asúa
}

Science in the Vanished Arcadia: Knowledge of Nature in the Jesuit Missions of

Paraguay and Río de la Plata. Leiden: Brill, 2014. Pp. xvi +385. Hb, 140.00 Euros.

Miguel de Asúa's study of Jesuit science in Paraguay during the seventeenth and eighteenth centuries adds to the growing literature on scientific production in the Spanish Americas. Most of the scientific works discussed by de Asúa were produced by Jesuits who lived and worked in the Guaraní missions of Paraguay, thereby linking his study to the on-going and vibrant research on this important colonial institution. While the missionaries' writings and scientific activities have long been recognized, de Asúa is the first to provide an in-depth study of the various types of scientific production they produced.

De Asúa structures Science in the Vanished Arcadia primarily based on different categories of scientific production: natural histories (chapter 1), medicine and herbals (chapter 2), cartography (chapter 3), and astronomy (chapter 4). Scholars focusing on any of these sciences will find the book a useful resource for identifying and tracking down primary source material. In each chapter, de Asúa introduces several Jesuits and describes the scientific work that they produced. Furthermore, de Asúa provides extensive background information about related works and detailed bibliographic and archival citations.

While de Asúa structures the book thematically, he also argues that the Jesuits' approach to science changed over time and varied based on individual producer. In the early years, Jesuit scholarship sought to explain and understand the foreign environment that they encountered, and as a result, Jesuit chronicles frequently referenced miracles and plants and animals with magical properties. De Asúa convincingly argues that not all Jesuit chronicles were full of such religious and natural wonders. As a more enlightened approach became popular, Jesuits progressively abandoned references to the supernatural. Still, the Jesuits' religious beliefs influenced their scholarship. Natural histories were organized following the sequence of the biblical story of creation and a motivation of advancing their religious goals underlay most all of the Jesuits' scientific works.

In contrast to scholarship that highlights innovation and advancement in the Jesuits' Spanish American economic endeavors, de Asúa asserts that Jesuit science was not in the vanguard of scientific thought. He claims that almost all of their scientific productions were expressions of Baroque culture and that only some of the productions of Jesuit exiles can be considered lesser examples of Enlightenment thought. As de Asúa shows, few of the Jesuits in Paraguay saw themselves as scientists, and for the most part, they were not motivated by 
the desire to advance science and scientific thought. De Asúa's detailed speculations about how individual Jesuit scholars copied from each other and/or built on previous works confirms that most were not concerned with making scientific advancements. Rather, they saw themselves as assembling informative guides for helping their confreres.

The Jesuits were motivated by utilitarian purposes associated primarily with their role as missionaries. They wrote natural histories and medicinal texts to provide useful information for advancing the missions, thereby enabling their order to save more souls. The identification of plants and animals by their aboriginal names reflected the Jesuits' prioritization of learning indigenous languages and facilitated communication with the Indians. Likewise, cartography, astronomy, and scientific experiments helped advance the Jesuits' evangelization efforts: maps informed fellow missionaries about the location of native peoples, natural resources, and trade routes; astronomy helped guide the missions' agricultural and liturgical calendar; and scientific experiments such as Ramón María Termeyer's work with spiders had the potential to advance and diversify the mission economy. More work remains to be done in exploring the economic and commercial motivations behind the Jesuits' scientific work. While utilitarian reasons clearly motivated much of the Jesuits' scientific production, de Asúa's claims about the pragmatic rationale behind astronomy are not as strong. Much of de Asúa's description of Jesuit astronomy deals with comets and eclipses without clearly explaining how such information was useful to the missionary effort. These studies and Jesuit correspondence with the European scientific community suggest that at least some Jesuits sought to contribute to advances in science.

De Asúa's analysis of the scientific works produced after the Jesuit expulsion (the focus of chapter 5 ) lacks much of the utilitarian rationale that permeates the rest of the book. An argument can be made that in this time period the Jesuits were motivated by a desire to defend their order and its activities in Paraguay. Their efforts to legitimize themselves and their knowledge based on first-hand exposure to the region suggest such a conclusion. For the most part, de Asúa's descriptions of the post-expulsion scientific production largely imply that the Jesuits wrote as a hobby or in an effort to contribute to science rather than to advance the Jesuits' missionary effort. This is especially true for Alonso Frías whose work has little to do with this study given that he was trained in Italy and conducted all of his research in Europe.

While de Asúa makes an effort to incorporate Guaraní influences, his study focuses primarily on the Jesuits and their motivations. Future scholarship will hopefully delve deeper into Guaraní beliefs and practices and how they influenced Jesuit science. This is certainly possible with the creative use 
of archival materials (letters, judicial proceedings, and other documents). Such information and linkages are not as readily apparent in the Jesuit scientific treatises and published primary sources upon which de Asúa bases most of his analysis. With its treatment of natural history, medicine, cartography, astronomy, and practical science, Science in the Vanished Arcadia provides a valuable overview Jesuit scientific production in Paraguay. It will most certainly be a valuable resource for scholars of Jesuit science and the Guaraní missions for years to come.

\section{Julia Sarreal}

Arizona State University

Julia.sarreal@asu.edu

DOI $10.1163 / 22141332-00201005-13$ 